\title{
Spectrochemical plasmas for clinical and biochemical analysis
}

\author{
Maria Montes-Bayón • Alfredo Sanz-Medel
}

Published online: 30 January 2015

(C) Springer-Verlag Berlin Heidelberg 2015

Plasma-based analytical strategies have been used in the context of elemental detection (total elemental determination) in biochemical and clinical samples for a number of years. But the applicability of these detection methods to more sophisticated analytical problems associated with the use, for instance, of new metallodrugs as therapeutic agents in different kinds of diseases or the interest in the fate of metallic nanoparticles in biochemical environments has reactivated the use of plasma-based methods in the bioworld. Thus, the state of the art of metal-derived information obtained in biological and clinical samples using plasma-based strategies has been the driving force for this special issue.

In this regard, from examination of the contributions to this issue, the first conclusion that can be drawn is that although element-specific detection can be conducted using a plethora of plasma techniques (a review on dielectric barrier discharge is included in this issue), inductively coupled plasma (ICP) mass spectrometry (MS) is still the most widely used detection method for bioapplications. The analytical advantages of ICP-MS over other elemental detection methods based on photon measurement (e.g., atomic absorption spectrometry or ICP optical emission

Published in the topical collection Spectrochemical Plasmas for Clinical and Biochemical Analysis with guest editors Alfredo Sanz-Medel and María Montes Bayón.

M. Montes-Bayón $(\triangle) \cdot$ A. Sanz-Medel

Department of Physical and Analytical Chemistry, Faculty of

Chemistry, University of Oviedo, C/ Julián Clavería 8,

33006 Oviedo, Spain

e-mail: montesmaria@uniovi.es

A. Sanz-Medel

e-mail: asm@uniovi.es spectrometry) are well known, and are mainly ascribed to the extremely high sensitivity of the technique together with its capability to measure isotopes and isotopic ratios, making possible screening and speciation of very low levels of heteroatoms in biomolecules or biological tissues. This fact has been exploited in some of the work reported in this issue for biosamples in combination with different separation methods necessary in order to achieve the desired species purity or directly on the tissues by applying laser ablation techniques.

Secondly, an interesting feature observed in the articles of this issue is the "quantitative" dimension of the work realized. Most articles contain quantitative elemental data and try to correlate them with species information (in the case of having these heteroelements as part of metabolites or proteins) or element/ drug distribution in tissues (imaging) by sorting with different quantification strategies. In this context, it is possible to address that plasma-based analysis becomes quantitative by providing a variety of strategies for absolute quantifications of low levels of the target heteroatoms in biological samples. In addition, it is noteworthy that the trend in some articles to combine the elemental information (provided by the corresponding plasma-based strategy) with specific biological informationfor example, protein activity or DNA-protein interaction - has been rarely addressed so far using ICP-MS. It may be that novel future uses of plasma technology go in this direction.

Lastly, regarding the topics of research chosen, it is possible to observe the increasing use of plasma-based strategies to address the fate of heteroelement-containing drugs in different biological samples or their consequences. It seems clear that the excellent analytical features provided by ICP-MS, in particular for monitoring platinumcontaining drugs of extended use in chemotherapy, have now been favorably used also for monitoring gadolinium 
contrast agents or boron-based therapies. Additionally, the monitoring of "nontraditional" plasma elements such as phosphorus has also been shown to play an important role, in particular for the monitoring of DNA-related samples, for which these techniques could play an important role soon. In this sense, the availability of the ICP-QQQ instrument, with considerably enhanced detection limits for the bioelements phosphorus and sulfur as compared with commoner ICP-MS instruments, could provide a substantial thrust for ICP-MS use in the genomics and proteomics fields.

In brief, we hope that this topical collection provides a flavor regarding the opportunities that plasma-based techniques are offering today and heralds their use in other possible challenging fields of research for clinical and biochemical analysis.

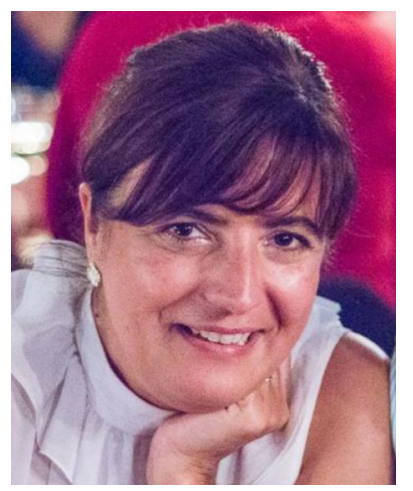

Maria Montes-Bayón has held a permanent position as Associate Professor in the Analytical Chemistry Area of the Department of Physical and Analytical Chemistry at the University of Oviedo since April 2008. She did her master degree at the University de Plymouth (UK) under the ERASMUS program supervised by Hywel Evans and Alfedo Sanz-Medel (November 1994). Immediately afterward, she started her PhD studies in Oviedo, and in July 1999 she defended her doctoral thesis, which was awarded the Extraordinary Prize in the Area of Analytical Chemistry by the University of Oviedo. In April 2000, she joined the research group of Joseph A. Caruso in the Department of Chemistry at the University of Cincinnati, Ohio (USA) as a Postdoctoral Fulbright Fellow until July 2002. In August of the same year, she returned to the University of Oviedo as a research scientist (Ramón y Cajal) in the Analytical Spectrometry Group and held this position until 2007. In 2008 she obtained the position of Associate Professor (accreditation as Full Professor in 2014). She is a coauthor of more than 100 original research publications and reviews, as well as several book chapters. She recived the Bunsen-Kirchhoff Award 2013 by the Working Group for Applied Spectroscopy in the Analytical Chemistry Division of the Gesellschaft Deutscher Chemiker (GDCh; German Chemical Society). She is member of the advisory boards of the Journal of Analytical Atomic Spectrometry and Metallomics, both published by the Royal Society of Chemistry.

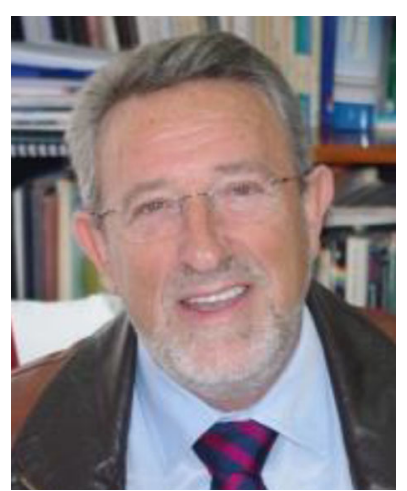

Alfredo Sanz-Medel has been a professor in the Department of Physical and Analytical Chemistry at Oviedo University (Spain) since 1982 . He is the author or coauthor of approximately 500 scientific publications in international journals, several patents, and books. His current research interests include new atomic detectors and ion sources for ultratrace analysis using plasmas, new molecular optical sensors, particularly those based on the use of quantum dots, and hybrid techniques, coupling a separation unit and an atomic detector, for ultratrace and trace metal speciation to solve biological and environmental problems and speciation for proteomics. This is aimed at integrating molecular (matrix-assisted laser desorption/ionization and electrospray mass spectrometry) and atomic (inductively coupled plasma mass spectrometry) mass spectrometry techniques, and the extensive use of inductively coupled plasma mass spectrometry techniques to perform heteroatom-tagged proteomics, both for qualitative and for quantitative purposes. He has been an editor of Analytical and Bioanalytical Chemistry since 2002. At Euroanalysis 2007, in Antwerp, he received the Robert Kellner Award. In 2011, he received the European Award for Plasma Spectrochemistry. 